\title{
Enfermedad de Hirschsprung en un adulto
}

\author{
Hirschsprung's Disease in an Adult Patient
}

Ramón Estopiñan Rebollar, MD, ${ }^{1}$ Ramón Estopiñan Cánovas, MD, ${ }^{1}$ Rafael Pila Peláez, MD. ${ }^{1}$

1 Hospital Provincial Clínico-Quirúrgico Manuel Ascunse Domenech. Camagüey, Cuba.

Fecha recibido: $09-12-14$ Fecha aceptado: 26-01-16

\begin{abstract}
Resumen
Objetivo: presentar un caso infrecuente de enfermedad de Hirschsprung en un adulto. Caso clínico: paciente masculino de 32 años de edad, con grave retraso intelectual y mental y con familiares de bajo nivel educacional. Tiene antecedentes de episodios de estreñimiento aliviados con laxantes desde la infancia; fue ingresado en múltiples ocasiones al hospital pediátrico de su provincia por el ya mencionado motivo. Se le diagnostica enfermedad de Hirschsprung mediante colon por enema y manometría rectal; de esta manera, se plantea una intervención quirúrgica como tratamiento pero no se cuenta con el permiso de los familiares. Acude al cuerpo de guardia del hospital refiriendo que llevaba 83 días sin defecar; en el examen físico presentaba abdomen globuloso, asimétrico, se palpaba una tumoración de $30 \mathrm{~cm} \times 15 \mathrm{~cm}$ en el hemiabdomen derecho. Tacto rectal: esfínter tónico, ampolla rectal vacía, ruidos hidroaéreos disminuidos. El estudio analítico fue normal. La radiografía de abdomen de pie y acostado mostró radiopacidad compatible con materia fecal. Se practica sigmoidectomía con colostomía tipo Hartman para resolver el cuadro agudo; el resultado quirúrgico demostró un megacolon agangliónico confirmado por histopatología. El paciente se encuentra asintomático en espera para tratamiento quirúrgico definitivo. Conclusiones: la enfermedad de Hirschsprung es rara en el adulto, y en estos casos debe diferenciarse de otras causas de megacolon. Su etiología es desconocida aunque se considera multifactorial; el primer y más importante síntoma es el estreñimiento, su diagnóstico se lleva a cabo con estudios imagenológicos y manometría rectal, y se confirma por histopatología. Su tratamiento siempre es quirúrgico.
\end{abstract}

\section{Palabras clave}

Enfermedad de Hirschsprung, adulto.

\begin{abstract}
Objective: This study presents a rare case of Hirschsprung's disease in an adult. Case Report: The patient was a 32 year old man with severe intellectual and mental retardation from a poorly educated family. Since childhood, he had had a history of episodes of constipation which was relieved with laxatives and for which he had been admitted on many occasions to the Children's Hospital of his province. Hirschsprung's disease was diagnosed with a barium enema and rectal manometry. Surgery was recommended, but his family did not give their permission. When he arrived at the hospital, he told doctors that he had not had a bowel movement for 83 days. Physical examination showed a globular, asymmetrical abdomen with a $30 \times 15 \mathrm{~cm}$ palpable tumor in the right abdomen. Digital rectal examination showed normal sphincter muscle tone and an empty rectal ampulla. Bowel sounds were minimal. The analytical study was normal. Standing and prone abdominal radiography showed x-ray opacity compatible with fecal matter. Hartmann's procedure was performed to resolve the acute situation. Surgery revealed aganglionic megacolon which was confirmed by histopathology. The patient was asymptomatic while awaiting definitive surgical treatment. Conclusions: Hirschsprung's disease is rare in adults, and must be distinguished from other causes of megacolon. Its etiology is unknown, although it is considered multifactorial. The first and most important symptom is constipation. Diagnosis requires imaging and rectal manometry and confirmation by histopathology. Treatment is always surgical.
\end{abstract}

\section{Keyword}

Adult Hirschsprung's disease. 


\section{INTRODUCCIÓN}

La enfermedad de Hirschsprung (EH) fue descrita por primera vez en 1888, en 2 lactantes, por el pediatra de Copenhague Herald Hirschsprung. Aunque poco frecuente ( 1 de cada 5000 nacidos), es una de las causas más comunes de obstrucción intestinal en el recién nacido (1).

La EH se considera como uno de los trastornos conocidos del disaganglionismo, que incluyen también el hipoganglionismo y la displasia neuronal intestinal (1). Se describe como la ausencia congénita de neuronas ganglionares en el plexo nervioso intestinal intrínseco, lo que condiciona la sobrecontracción del segmento afectado (2). Es una enfermedad genética relativamente frecuente pero de transmisión compleja, por lo que el mendelismo no es siempre aparente. Actualmente, se han establecido las bases patogénicas y los aspectos genéticos de la enfermedad; entre ellos se han descrito alrededor de 13 genes involucrados en la morfogénesis y la diferenciación del sistema nervioso entérico (3). Hasta ahora, las mutaciones más importantes son las del gen RET (receptor transmembrana de la tirosinasa), situado en el brazo largo del cromosoma 10 , que se detectan hasta en un $50 \%$ de los casos familiares de $\mathrm{EH}$ y hasta en un $20 \%$ de los casos esporádicos, estando más relacionados con el trastorno de segmento largo (3).

\section{CASO CLÍNICO}

Paciente masculino de 32 años de edad, con grave retraso intelectual y mental y con familiares de bajo nivel educacional, campesinos, provenientes de otra provincia (Las Tunas). Tiene antecedentes de episodios de estreñimiento aliviados con laxantes desde la infancia. Fue ingresado en múltiples ocasiones al Hospital Pediátrico de Las Tunas por estreñimiento que no respondía positivamente al uso de laxantes ni enemas evacuantes; se le diagnostica enfermedad de Hirschsprung mediante colon por enema y manometría rectal pero los familiares se oponen a la intervención quirúrgica como tratamiento. Ahora acude al cuerpo de guardia del hospital refiriendo que llevaba 83 días sin defecar; presenta dolor abdominal difuso tipo cólico, así como un síndrome constitucional importante.

El examen físico, regional y por aparatos y sistemas fue normal, excepto por desnutrición moderada, con tensión arterial de $110 / 70 \mathrm{mmHg}$ y frecuencia cardíaca central de 112 latidos por minuto; presentaba abdomen globuloso asimétricamente que sigue los movimientos respiratorios, ruidos hidroaéreos disminuidos, doloroso difusamente donde se palpa una tumoración de $30 \mathrm{~cm}$ de largo y $15 \mathrm{~cm}$ de ancho que ocupa aproximadamente el hemiabdomen derecho, de bordes regulares, superficie lisa y más bien blanda. Red venosa abundante y visible. En el tacto rectal presentó esfínter tónico, ampolla rectal vacía, no se palpó tumor ni hay dolor al examen. El estudio analítico fue normal en hemoquímica, enzimas, iones y estudio de la función renal y pancreática. La radiografía de tórax mostró elevación de ambos hemidiafragmas; en las radiografías de abdomen simple de pie, acostado, anteroposterior y lateral se observa abdomen blando, ocupado con radiopacidad que impresiona la materia fecal hacia el hipocondrio y el flanco derecho, con asas intestinales dilatadas. En el ECG se observa taquicardia sinusal.

$\mathrm{Al}$ tener en cuenta el diagnóstico de $\mathrm{EH}$, se trata de realizar una intervención quirúrgica, como es compatible en estos casos, pero los familiares se oponen; se realiza tratamiento quirúrgico urgente, donde se practica sigmoidectomía con colostomía (figuras 1 y 2) de tipo Hartman, en aras de resolver el cuadro agudo. Se encontró gran distención del colon ocupado por materia fecal hasta el tercio superior del recto, donde se halló un área de estenosis que impedía el paso del contenido fecal; se envía la pieza quirúrgica para estudio histopatológico. Se describe segmento intestinal de $60 \mathrm{~cm} \times 25 \mathrm{~cm} \times 15 \mathrm{~cm}$ exageradamente distendido y con la apertura ocupada totalmente por heces fecales compactas, se presenta distensión severa y borramiento total de los pliegues de las paredes acartonadas (figura 3). El diagnóstico histológico es compatible con megacolon agangliónico (figura 4).

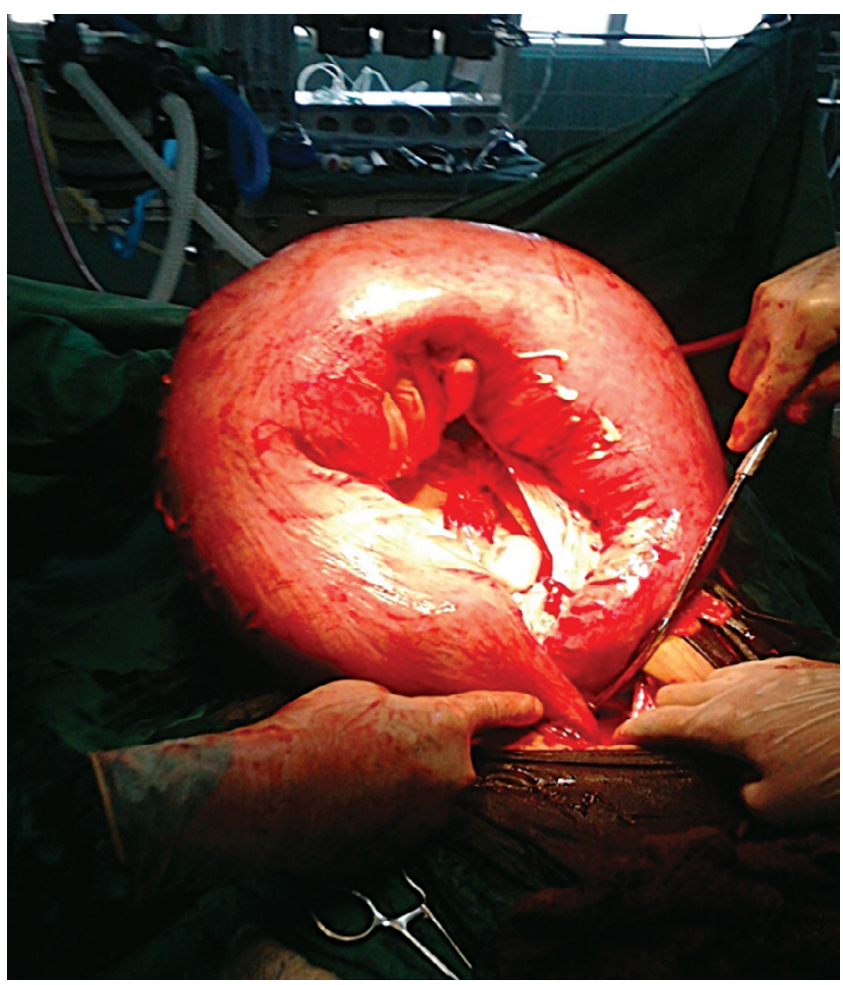

Figura 1. Distención del colon durante el acto quirúrgico en un paciente con $\mathrm{EH}$. 


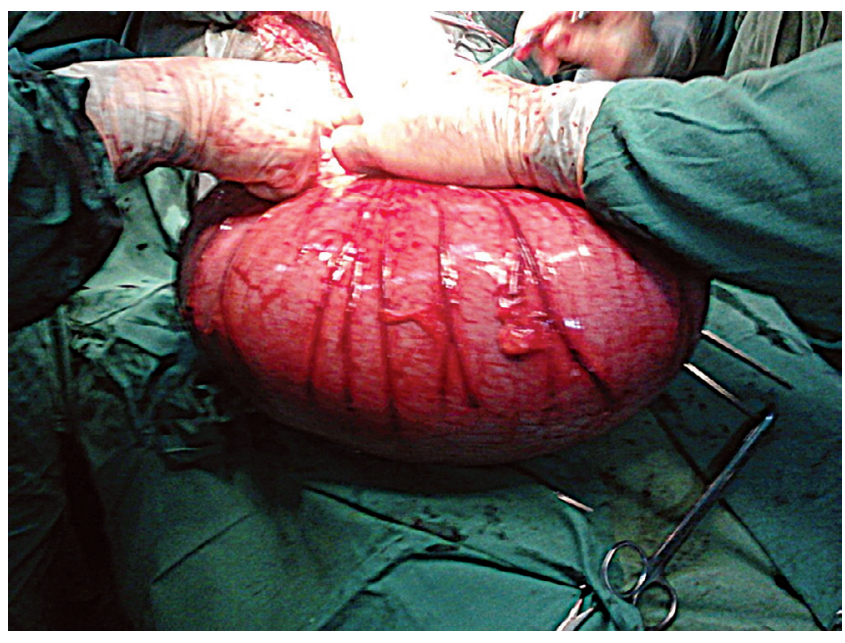

Figura 2. Aspecto del colon durante la terminación de la intervención quirúrgica.

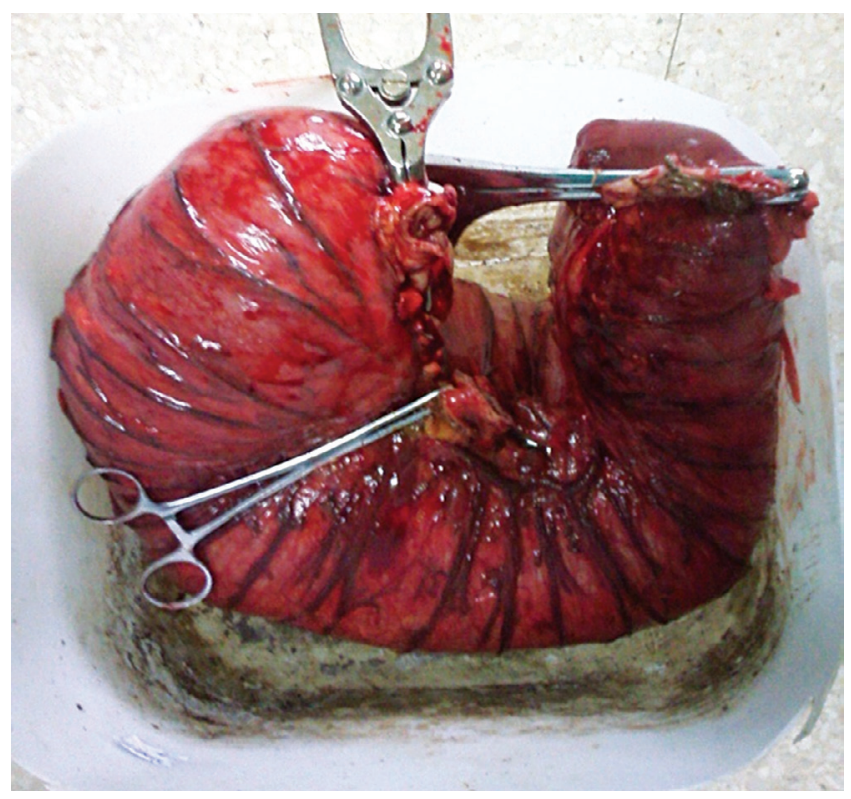

Figura 3. Pieza quirúrgica disecada de $60 \mathrm{~cm} \times 25 \mathrm{~cm} \times 15 \mathrm{~cm}$.

Con los anticuerpos para calretinina se confirma la enfermedad de Hirschsprung por la ausencia de células ganglionares y la hipertrofia del plexo nervioso intramural.

El paciente se atiende en consulta externa de coloproctología para tratamiento definitivo y ahora se encuentra asintomático.

\section{DISCUSIÓN}

El límite inferior del aganglionismo, el esfínter anal interno, es constante; el límite superior es variable y sirve para clasificar la enfermedad. La EH más frecuente es la de segmento corto, donde el aganglionismo no se extiende más allá del

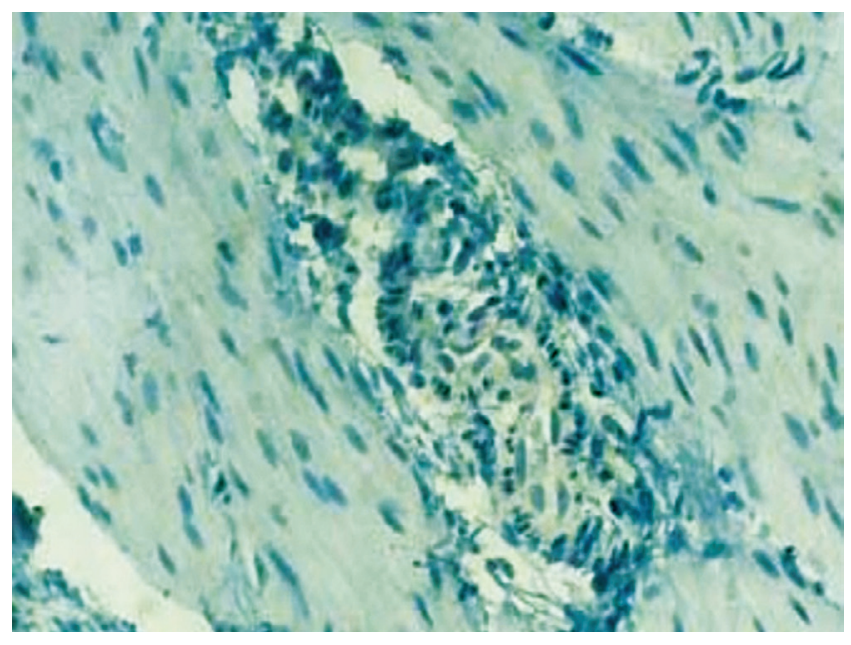

Figura 4. Histopatología.

sigma ( $80 \%$ de los casos); un pequeño número de esta clase es de segmento ultracorto o yuxtanales, cuando ocupa solo el recto distal, por debajo del suelo de la pelvis. El segmento largo se presenta cuando el aganglionismo es proximal al sigma (20\%); en una minoría de este grupo, el segmento ocupa todo el colon (EH colónica) o todo el intestino (EH intestinal) (4). Este caso se trataría de una $\mathrm{EH}$ de segmento corto, que ocurre en forma aislada en el $70 \%$ de los casos; en el 30\% aparece en compañía de otras anomalías congénitas (2); en un $12 \%$ existe una cromosomopatía, casi siempre síndrome de Down, y en el otro $18 \%$ habría otras alteraciones sindrómicas o distintas alteraciones aisladas (2). Este caso presentaba un retardo mental que no se trató por negativa familiar.

La etiología es desconocida aunque se considera multifactorial (1). Los síntomas varían según la edad, la extensión del área afectada y la presencia de complicaciones; la longitud del segmento afectado no está relacionada con la edad de presentación ni con la gravedad de los síntomas (3). El síntoma principal es el estreñimiento de aparición temprana en los recién nacidos a término (3); los recién nacidos y lactantes pequeños casi siempre presentan clínica de obstrucción intestinal (3). El estreñimiento intermitente (en el $66 \%$ de los pacientes) se inicia en el periodo de neonato; puede ocurrir distención abdominal y vómitos. $\mathrm{Si}$ el estreñimiento no tiene una causa mecánica (atresia intestinal, íleo meconial, malrotación o ano imperforado) debe pensarse en un EH (5).

Se puede encontrar, como en este paciente, una gran dilatación abdominal, con red venosa visible, distención, anorexia, vómito e incluso retraso del crecimiento (4). Su diagnóstico en la edad adulta probablemente corresponda a causas congénitas no detectadas previamente (5\%) o pacientes mal diagnosticados de estreñimiento crónico, 
y suele realizarse tras alguna complicación, como la volvulación intestinal $(3,4)$. El estudio radiológico simple y contrastado, en posición anteroposterior y lateral, permite apoyar el diagnóstico cuando hay un segmento estenosado (agangliónico), generalmente de recto o rectosigmoide (80\%-85\% de los casos) (6). La mayoría de los autores (5), no todos (6), plantean que antes de practicar la cirugía correctiva debe tenerse la certeza del diagnóstico positivo, que se establece solo con la biopsia de la pared rectal, la cual tiene una sensibilidad y una especificidad cercana a $100 \%$ (6). Histológicamente, la EH se caracteriza por la ausencia de células ganglionares parasimpáticas, acompañada frecuentemente por hipertrofia de los troncos nerviosos (69\%) (7), como en este caso.

Con el advenimiento de la inmunohistoquímica como técnica en el diagnóstico histopatológico, se han propuesto varios anticuerpos que permiten identificar con certeza células ganglionares; entre los marcadores que han demostrado mayor utilidad están los anticuerpos para calretinina, con los que se alcanzan sensibilidad y especificidad de $100 \%$ (6, 7 ), aunque no está presente en nuestro medio. El colon por enema ofrece más información que la radiografía simple, sobre todo en pacientes no agudos, y aunque su sensibilidad (70\%) y especificidad ( $83 \%$ ) son inferiores a otras pruebas diagnósticas, suele ser la primera que se realiza (7).

El estudio practicado a este paciente en el hospital y que ofreció el diagnóstico fue la manometría rectal, que tiene alta sensibilidad ( $91 \%$ ) y especificidad ( $94 \%$ ) y resulta muy útil en los casos de segmento agangliónico ultra corto (2). No obstante, el diagnóstico de certeza se realiza por estudio histológico, aunque la técnica considerada como patrón de oro es la biopsia quirúrgica, que incluye capa muscular (sensibilidad y especificidad $100 \%$ ) y que habitualmente se realiza por succión; además de disminuir complicaciones (infección, sangrado, perforación), evita la anestesia general y las suturas (6).

Entre los casos de megacolon en el adulto para tener en cuenta está $\mathrm{EH}$, displasia neuronal intestinal, megacolon adquirido, enfermedad de chagas secundaria de estreñimiento, pseudobstrucción intestinal crónica, megacolon tóxico por infección, inflamatorio, entre otros $(3,5)$.

Una vez se confirma el diagnóstico, el tratamiento siempre es quirúrgico (8); la resección quirúrgica del segmento afectado consigue resultados favorables en la mejoría de los pacientes (8). En este caso, después de practicar sigmoi- dectomía con colostomía (tipo Hartman) de urgencias, el paciente se prepara para tratamiento definitivo.

\section{CONCLUSIONES}

La EH o megacolon agangliónico en el adulto es un trastorno motor intestinal resultado de la aganglionosis congénita del colon, propia de edades pediátricas, que produce finalmente una oclusión funcional intestinal. Su diagnóstico en pacientes adultos es muy poco frecuente, pudiendo causar múltiples complicaciones y diagnósticos erróneos si no se tiene en cuenta sus antecedentes.

\section{Conflicto de intereses}

Los autores no tienen conflictos potenciales de intereses en este trabajo.

\section{REFERENCIAS}

1. Menchaca Cervantes C, Rodriguez Velazco A, Ramón García G, Rendom Macías ME. Enfermedad de Hirschsprung. La inmunohistoquímica como apoyo para el diagnóstico. Rev Med Inst Mex Seguro SOE. 2013;51(6):610- 3.

2. Hart J, Wilcox R, Weber C. The gastrointestinal tract. Ed: Stockes JT, Dehner LP, editors. Pediatric pathology. Third edition. USA: wolters kluwer/Lippincott Williams and wilkins; 2011 p 592- 96.

3. Yuksel I, Ataseven I, Ertugrul L, Basar O, Sasmaz N. Adult segmental Hirschprung's disease. South Med J. 2009; 102:184- 5 .

4. Fortea C, Martinez D, Rivadulla I, Daroca J, Paira Gitol. Hirschsprung's disease in adults. Rev Esp Enferm Dig. 2001;3:150-151.

5. Haricharan RN, Georgeson KE. Hirschsprung's disease. Senein Pediatr Surg. 2008;17:266-75.

6. Kapur RP, Reed RC, Tinn LS, Patterson K, Joanson J, Rothedge JC. Calretinin inmunohistochemestry in the evaluation of suction rectal biopsies for Hirschsprung's disease. Pediatr Dev Pathol. 2009;12(1):6-15.

7. Memarzadeh M, Talebi A, Edalaty M, Hosseinpoor M, Vattidi N. Hirschsprung's disease diagnosis: Comparison of inmunohistochimical, hematoxilin and eosin staining. J Indian Assoc Pediatr Surg. 2009;14(2):59-62.

8. Dasgupta R, Langer J. Evaluation and management of persistent problems after surgery for Hirschsprung's disease in a child. J Pediatr Gastrointernal. 2008;46:13-19. 